\title{
Supercluster-Coupled Crystal Growth in Metallic Glass Forming Liquids
}

Yujun Xie ${ }^{1,2}$, Sungwoo Sohn ${ }^{1}$, Minglei Wang ${ }^{1}$, Huolin Xin ${ }^{3}$, Yeonwoong Jung ${ }^{4}$, Mark D. Shattuck ${ }^{5}$, Corey S. O’Hern ${ }^{1}$, Jan Schroers ${ }^{1}$, Judy J. Cha ${ }^{1,2^{*}}$

1. Department of Mechanical Engineering and Materials Science, Yale University, New Haven, USA

2. Energy Sciences Institute, Yale West Campus, West Haven, USA

3. Center for Functional Nanomaterials, Brookhaven National Laboratory, Upton, USA

4. Department of Materials Science and Engineering, University of Central Florida, Orlando, USA

5. Department of Physics and Benjamin Levich Institute, City College of the City University of New York, New York, USA

* Corresponding author: judy.cha@yale.edu

Mechanisms of crystallization in solids are ubiquitously important in determining materials properties, yet their near atomistic understanding is incomplete due to the lack of appropriate materials systems and direct experimental tools to probe the crystallization processes in detail. Metallic glasses (MGs) possess simple metallic bonds and slow crystallization kinetics, making them uniquely suitable to study crystallization. We have been directly investigating crystallization of MG forming liquids by in situ heating sizecontrolled MG nanorods down to $\sim 5 \mathrm{~nm}$ inside a transmission electron microscope [1-4].

Using MG nanorods, we have discovered several crystallization phenomena at the nanoscale, which deviate greatly from the expected, macroscopic behaviors. These include the non-monotonic crystallization kinetics as a function of the diameter of the rod [1], the merging of the critical heating and critical cooling rates for very small nanorods [2], and the single crystalline grain out of multi-component MG alloys at the nanoscale instead of the expected poly-crystalline multiple grains [2]. The pronounced deviations in the observed crystallization phenomena at the nanoscale allow us to test the limits of classical theories and open possibilities to develop atomistic understanding of nucleation and crystallization.

In this talk, we extend our crystallization studies of MG nanorods and investigate the growth dynamics after the nucleation event. We conduct atomically-resolved in situ isothermal crystallization experiments on MG nanorods [4]. We find that the common growth models, which assume a structure-less liquid at the interface of a growing crystalline solid and the liquid, fail to explain our in situ TEM experiments. Instead, we find that the crystal growth kinetics depends strongly on the local ordering that is present in the glass. The key experimental finding is this: At the same crystallization temperature, we observe that the crystal growth rate of a heated metallic glass is much higher than that of a cooled metallic liquid. For liquid metals, structural ordering has been shown. Thus, our hypothesis is that the structural ordering enhances crystal growth, in contrast to assumptions from common growth models. The asymmetric growth rates are attributed to the difference in the population of the local ordering, which is present in the glass state while largely absent in the melt state.

Direct visualization reveals the structural ordering as densely populated small clusters in a nanorod that is heated from the glass state, and similar behavior is found in molecular dynamics simulations of model metallic glasses. The structural ordering can be controlled by the thermal history of the MG rods, and we observe that the crystal growth kinetics changes with the thermal treatment of the MG rods. The present results demonstrate that classical growth models are inadequate for describing crystallization of most metallic alloys. 
[1] S. W. Sohn*, Y. Jung*, et al., Nat. Commun. 6 (2015), 8157.

[2] S. W. Sohn*, Y. Xie*, et al., Nat. Commun. 8 (2017), 1980.

[3] Y. Xie, et. al., JOM 69 (2017), p.2187-2191.

[4] Y. Xie*, S. W. Sohn*, et al., Nat. Commun. 10 (2019) 915.

(* denote equal contributions.)

a)

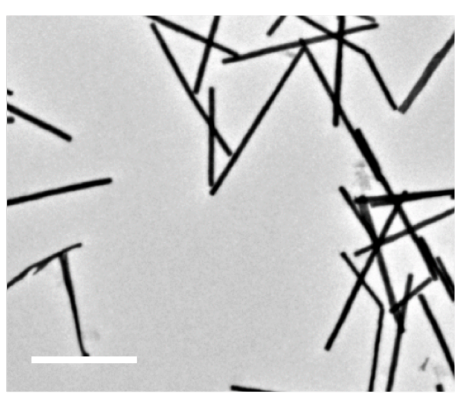

c)
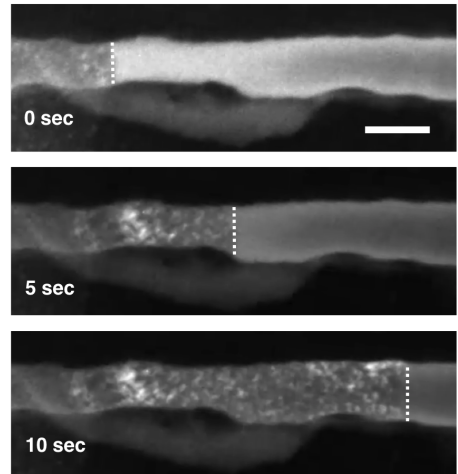

b)

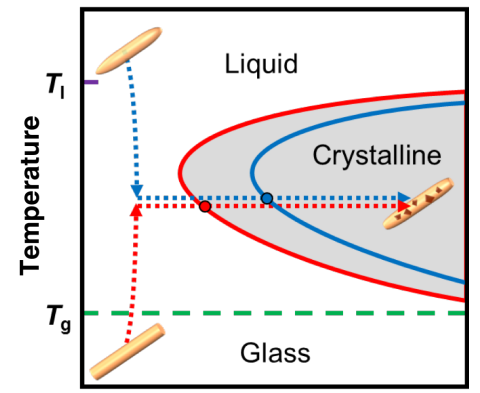

d)
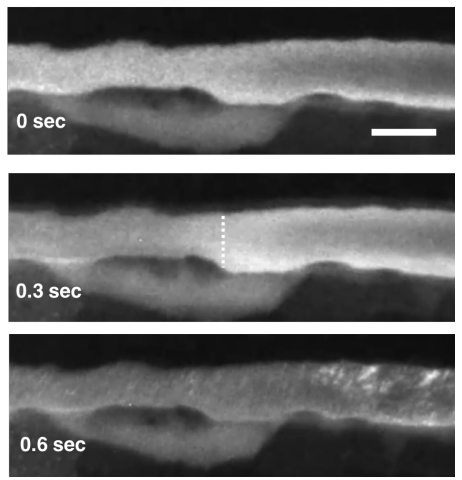

Figure 1. (a) MG nanorods. Scale bar $=1$ micron. (b) Two paths for isothermal crystallizations (blue and red dotted lines). (c) Snapshot dark field TEM images of a MG rod undergoing crystallization, cooled rapidly from $900^{\circ} \mathrm{C}$ (liquid) to $420^{\circ} \mathrm{C}$. (d) Snapshot dark field TEM images of the same MG rod undergoing crystallization, this time heated rapidly from room temperature (glass) to $420^{\circ} \mathrm{C}$. Scale bar $=80 \mathrm{~nm}$. Growth kinetics is much faster for the heated case.

a)

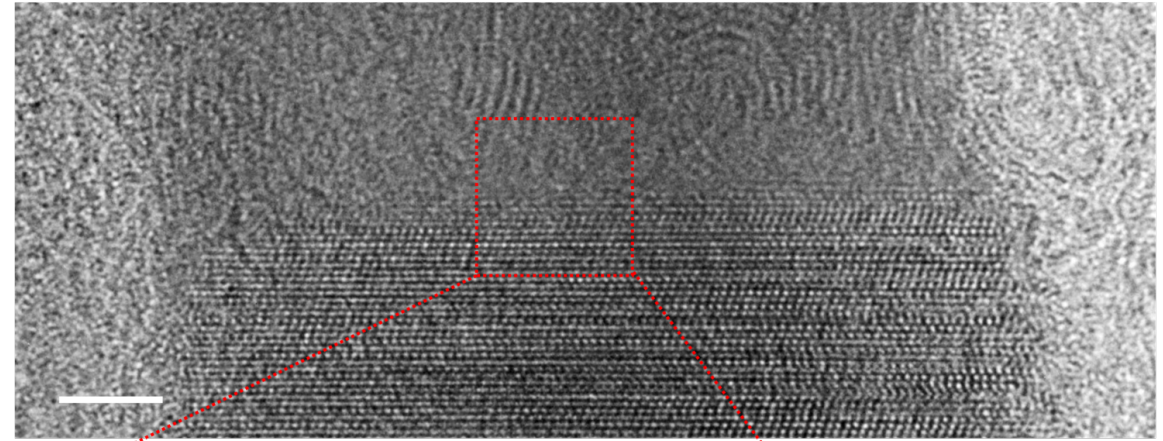

b)

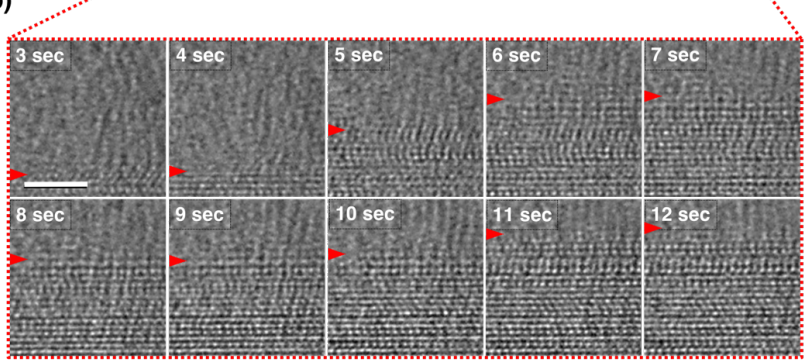

c)

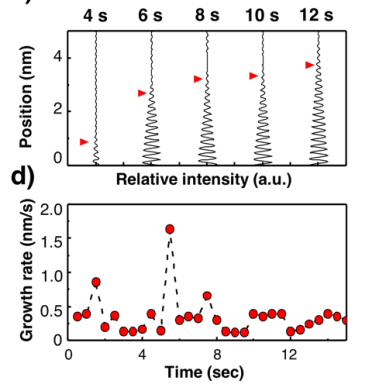

Figure 2. (a) A snapshot from an in situ TEM movie of a $23 \mathrm{~nm} \mathrm{MG}$ rod upon rapid heating from room temperature to $360^{\circ} \mathrm{C}$. (b) Zoomed-in snapshots with 1 second time intervals from the region marked with a red box. The growth front is marked by the red arrow. Scale bars $=2 \mathrm{~nm}$. (c) Intensity profiles from the image to mark the growth position. (d) Measured growth rate as a function of time. 